\title{
Studying oxygen vacancies in ceramics by perturbed angular correlation spectroscopy
}

\author{
Han-Tzong Su, Ruiping Wang, Heinz Fuchs, John A. Gardner \\ Department of Physics, Oregon Sitate University, Corvallis, OR 97331-6507 \\ William E. Evenson \\ Department of Physics, Brigham Young Úniversity, Provo, UT 84602 \\ Janzes A. Sommers \\ Teledyne Wah Chang, Inc., Albany, OR 97321
}

\begin{abstract}
Ferturbed angular correlation measurements in tetragonal and cubic zirconia and in ceria are described. A physically reasonable and self-consistent interpretation of these data implies that oxygen vacancies are trapped at a second neighbor position by $C d$ in tetragonal zirconia and by $I n$ in ceria. For $C d$ in tetragonal zirconia, the vacancy trap energy is found to be $0.44 \mathrm{eV}$, and the energy barrier between adjacent trap sites is approximately $0.8 \mathrm{eV}$. The activation energy of an oxygen vacancy hopping between trap sites around ${ }^{111} \mathrm{Cd}$ in ceria is found to be $0.55 \mathrm{eV}$. The activation energy for oxygen vacancy hopping in cubic zirconia, as detected by ${ }^{181} \mathrm{Ta}$ $\mathrm{PAC}$, is about $1.0 \mathrm{eV}$ and independent of the $Y$ concentration.
\end{abstract}

Key Words: Zirconia, Ceria, Oxygen vacancies, Oxygen vacancy hopping energies, Oxygen vacancy trap energies

\section{DISCLAIMER}

This report was prepared as an account of work sponsored by an agency of the United States Government. Neither the United States Government nor any agency thereof, nor any of their employees, makes any warranty, express or implied, or assumes any legal liability or responsibility for the accuracy, completeness, or usefulness of any information, apparatus, product, or process disclosed, or represents that its use would not infringe privately owned rights Reference herein to any specific commercial product, process, or service by trade name, trademark, manufacturer, or otherwise does not necessarily constitute or imply its endorsement, recommendation, or favoring by the United Siztec Government or any agency thereof. The views and opinions of authors expressed herein do not necessarily state or reflect those of the United States Government or any agency thereof.

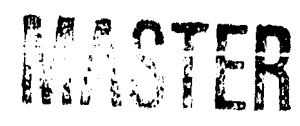

DSTRIBUTION OF THIS DOCUMENT IS UMLIMITED

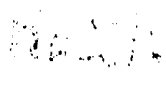

(1) 


\section{Introduction}

Experimental methods utilizing nuclear interactions provide powerful tools for studying the local structure, magnetic interactions, defect-complexing, and microscopic dynamic structural properties of condensed matter. Among these techniques are nuclear magnetic resonance (NMR), Nuclear quadrupole resonance (NQR), Mossbauer, and perturbed angular correlation (PAC) spectroscopies. In this paper we describe recent PAC research into the properties of oxygen vacancies in doped zirconia and ceria ceramics.

Time differential gamma-gamma PAC was employed for this work $[1,2]$. These experiments require that a radioactive element be introduced into the sample. The nucleus decays by emitting two gamma rays whose emission directions are correlated. The experiment detects the perturbation of the angular correlation by magnetic or electric fields acting on the nucleus during the time period between the emission of the two gamma rays. For the work discussed here, the interaction between the nucleis electric quadrupole moment and electric field gradients (efgs) due to nearby oxygen vacancies is probed at either a ${ }^{111} \mathrm{Cd}$ or a ${ }^{181} \mathrm{Ta}$ nucleus. These nuclei are the daughter nuclei of the ${ }^{111}$ In or ${ }^{181} \mathrm{H} f$ radioactive parent isotopes that are originally introduced into the samples. The physical information, i.e. the efg, is the same 
quantity measured in an NQR experiment, but the sensitivity of the methods is very different. In the work reported here, we exploit two important advantages of PAC over NQR. These advantages are the temperature-independent sensitivity of PAC and its intrinsic capability of detecting rapidly relaxing or broadly-distributed electric field gradients.

We report here studies of ceria and two forms of zirconia. All of our ceria samples are in the cubic fluorite structure. We have studied pure and lightly-doped zirconia in its tetragonal symmetry polymorph, which is stable at temperatures greater than about $1000^{\circ} \mathrm{C}$, as discussed below. We have also studied yttria-stabilized cubic zirconia, with greater than about $15 \%$ yittrium, which is stable at all temperatures.

The efg is produced by ions neighboring the PAC probe atom. We describe in this paper experiments where that efg is strongly influenced by oxygen vacancies. At low temperature the experiments detect a static efg from which we determine the approximate distribution of defects around the probe atom. For both PAC probes used here, ${ }^{111}$ In and ${ }^{181} \mathrm{Hf}$, the daughter isomeric states following radioactive decay to ${ }^{111} \mathrm{Cd}$ and ${ }^{181} \mathrm{Ta}$, respectively, are short lived. Therefore, the chemistry of the probe/vacancy interaction is determined by the parent isotope at low temperature, but the efg re- 
flects the local lattice relaxation around the daughter nucleus. At high temperature, oxygen vacancies move rapidly, and the experiment detects both a time-averaged efg and some information about its time-dependence. In this regime, the experiment measures equilibrium properties of the daughter atom. These data allow us, in principle, to deduce the energy and location of the deepest vacancy trap adjacent to both $I n$ and $C d$ as well as the activation energy barrier between adjacent equivalent trap sites around $C d$. With ${ }^{181} \mathrm{Ta}$ probes in cubic yttria-stabilized zirconia, the efg fluctuates at a rate which depends on both the concentration and the average hopping energy barrier of oxygen vacancies. These fluctuations produce relaxation in the PAC spectrum which reflects these dynamic properties of the oxygen vacancies.

\section{PAC Overview}

Technical details of the PAC spectrometer and analytic procedures have been described elsewhere[1,3,4] and will not be repeated here. We will only review briefly the physical concepts. The energy levels of both the ${ }^{111} C d$ and ${ }^{181} \mathrm{~T} a$ intermediate states probed by PAC have spin $\mathrm{I}=5 / 2$ and are split into three doubly-degenerate levels by an efg[5,2]. If the efg is static and identical for all nuclei, then a PAC experiment is characterized by three well- 
defined frequencies, $\omega_{1}, \omega_{2}$, and $\omega_{3}=\omega_{1}+\omega_{2}$. In general $1 \leq \omega_{2} / \omega_{1} \leq 2$. The relationship between the frequencies and the nuclear energy level splitting is shown in Fig. 1. The figure shows a typical "ideal" spectrum, that of ${ }^{111} \mathrm{Cd}$ in ceria at $150^{\circ} \mathrm{C}$. The figure also contains a schematic representation of the local potential energy distortion by a vacancy, which is believed to be responsible for the observed efg.

Such ideal spectra are the exception rather than the rule. In insulators, one often observes strong poorly-understood "after-effects" of the nuclear decay from the parent isotope. We observe after-effects in monoclinic zirconia and $N b$-doped ceria. We shall not discuss such data here and limit all further discussion to cases where after-effects play no role.

At sufficiently low temperature, an efg interaction will be static on the PAC time scale $(<1 \mu s)$. However, impurities and structural defects usually cause the efg to vary somewhat from site to site. If this variation is small, it can usually be approximated adequately by a Lorentzian frequencybroadening term incorporated into the computer fitting routine.

At higher temperature in the oxides of interest here, oxygen vacancies hop rapidly, and the efg fluctuates significantly between the emission of the two gamma rays. There is no generally-applicable way to model such time- 
dependent effects analytically. We have used a stochastic model $[6,7]$ to predict PAC spectra for cases where the dominant dynamic contribution to the efg arises from trapped mobile defects such as oxygen vacancies trapped by lower-valent cation dopants in zirconia and ceria. We believe this model to be an excellent approximation for the ${ }^{111} C d$ PAC data reported here. The model also describes properly the dynamic effects detected in the highertemperature ${ }^{181} \mathrm{Ta}$ PAC experiments on cubic yttria-stabilized zirconia.

It should be emphasized that the interpretation of these data (and, in fact, almost any hyperfine experimental data) in terms of physical quantities such as vacancy trap and hoppin ${ }^{5}$ barrier energies is not an unambiguous, straightforward procedure. Computer fits to hyperfine spectra involve many parameters, and it is not mathematically difficult to achieve an excellent fit that is physically meaningless. In this work we have used only physicallymotivated models whose spectral parameters are related directly to the vacancy/nucleus interactions and to microscopic parameters describing the defect motion. Most information is derived from parametric dependence on temperature and composition, since fits become essentially unique when a single model is required to be consistent with spectra representing a wide range of temperatures and compositions. Technical details of the model and 
fitting procedures $[7,8]$ are described elsewhere.

\section{Experimental Procedure and Analysis}

\section{A. Samples and Procedure}

The number of PAC spectra that can be measured for a given sample is limited by the half-life of the parent isotope. An accumulation time of 24 hours is typically required to obtain spectra with sufficient statistical accuracy. Samples using ${ }^{111}$ In as the radiotracer can be used for approximately 10 days before the activity of the 2.8-day-half -life isotope decays to an unusably low level. ${ }^{181} \mathrm{Hf}$ has a 42 day half-life, so samples utilizing this nucleus as the probe can be used for several months.

All samples were made by coprecipitation from high-purity oxychloride or nitrate solutions. Samples for ${ }^{111}$ In/Cd PAC experiments were made by adding the desired activity, typically a few microCuries, of the ${ }^{111}$ In radiotracer prior to precipitation. ${ }^{111} \mathrm{In}$ is obiained in $\mathrm{HCl}$ or $\mathrm{HNO}_{3}$ solution from Dupont/New England Nuclear Corporation. For ${ }^{181} \mathrm{H} f / T a$ PAC experiments, all samples reported here were made from zirconium from which the naturally-occuring hafnium had not been removed. The samples were irradiated in the Oregon State University Tri ${ }^{a}$ reactor to produce the desired level of ${ }^{181} \mathrm{Hf}$ activity. 
${ }^{11} C d$ PAC experiments are reported on tetragonal zirconia in a temperature range 1000 to $1400^{\circ} \mathrm{C}$. Data were first accumulated at $1400^{\circ} \mathrm{C}$, and then at lower temperature. Once transformed to tetragonal symmetry, the samples were held at temperatures high enough to prevent retransformation to the monoclinic phase. All samples remained tetragonal down to $1000^{\circ} \mathrm{C}$. Samples of pure zirconia, and $Z r_{1-x} M_{x}$ oxide with $M=N b, Y$, or In and $x$ from 0.001 to 0.02 were measured. These cation additions were made to control the oxygen vacancy concentration. The ${ }^{111}$ In concentrations were trace level (of order $10^{-8}$ ).

The ceria samples were subjected to a 6 -hour $1600^{\circ} \mathrm{C}$ heat treatment to produce grains of order $10 \mu$ dimension. In smaller grains, a significant fraction of the indium tracer atoms apparently were concentrated in interface regions not representative of bulk material. After this heat treatment, PAC data were accumulated over a temperature range of $77 \mathrm{~K}$ to $1200^{\circ} \mathrm{C}$. These spectra were independent of thermal history. Yttria-stabilized zirconia samples were annealed at $1400^{\circ} \mathrm{C}$ prior to data accumulation. Lower temperature heat treatment did not produce homogeneous samples. Only data accumulated above $700^{\circ} \mathrm{C}$ are reported. These spectra are independent of thermal history provided the initial annealing period is sufficient to assure 
homogeneity.

The ceria sample stock solutions contain approximately 300 ppm of rareearth impurities. The content of other cation impurities is less than $50 \mathrm{ppm}$. The PAC data indicate that oxygen vacancies are present in the undoped ceria samples. These may be due to the rare-earth impurities whose ionic charge is compensated by oxygen vacancies.

The concentration of impurities ir the $Z r_{1-x} M_{x}$ oxide sample solutions is less than $100 \mathrm{ppm}$ apart from the approximately 1 at. \% level of $H f$ in the yttria-stabilized cubic zirconia samples.

The PAC data indicate presence of an oxygen-vacancy concentration approximately $350 \mathrm{ppm}$ larger than that required for charge compensation of dopants in the tetragonal zirconia samples. We cannot presently explain the origin of these additional vacancies. It is possible that they arise because of accidental aluminum contamination of samples by the alumina sample holder. Chemical analysis indicates presence of aluminum in samples aft. measurement, but there is good reason to believe that this is due to alumina contamination and not to aluminum incorporation into the zirconia. The additional vacancies may arise from structural defects or other mechanisms unrelated to impurity compensation. They are certainly not due to thermal 
generation of vacancy/interstitial pairs, whose concentration at these temperatures is negligible. We suspect that the additional vacancies arise from structural defects and plan further experiments to test this possibility.

B. Analysis of the PAC Spectra

Effects of oxygen vacancy motion on PAC spectra have been modeled by assuming that the efg fluctuates randomly among a small number of welldefined values. The form of the PAC spectrum is $\mathrm{cc}$. Iputed exactly for any given model[7]. The only serious uncertainty in fitting these PAC spectra is the electric field gradient distribution in alloys. Present models are too crude to provide a quantitative description of the broad efg distribution in the cubic zirconia samples. Fortunately, the nuclear relaxation caused by vacancy motion can be deduced reliably, and we limit our discussion to this important parameter and its physical implications.

The efg distribution is modeled adequately by a simple Lorentzian approximation for the very dilute tetragonal $Z r_{1-x} M_{x}$ oxide alloys - those with $x \leq 0.003$. The PAC spectral line-broadening for larger $x$ is large enough to reduce the quality of the spectral fits. In particular, the accuracy of the fitted relaxation parameter $\lambda$ is uncertain for larger $x$. The fundamental frequency $\omega_{1}$, for ${ }^{111} \mathrm{Cd}$ in the tetragonal $Z_{r_{1-x}} M_{x}$ oxides is shown in Fig. 
2. For small $x, \omega_{2} / \omega_{1}=2$, and this ratio decreases somewhat for larger $x$, as expected for an axially-symmetric electric field gradient broadened by random interactions with the $M$ dopants. The data shown in Fig. 2 were not strongly sensitive to the approximation used to account for frequency broadening. The total experimental uncertainty is no larger than the size of the symbols. The relaxation parameter is more sensitive to the broadening approximation, and we will not discuss these data in detail here.

The ${ }^{111} C d$ PAC spectrum for ceria shown in Fig. 1 is typical of spectra below $300^{\circ} \mathrm{C}$. At higher temperature the spectra are qualitatively different, having a time-averaged efg of zero and significant relaxation. $\lambda$ is shown for these temperatures in Fig. 3. We expect our spectral model to be excellent for all these data and find that the fits match the data ideally within statistical accuracy.

In Fig. 4(a), we show the $\lambda$ relaxation parameter affecting ${ }^{181} \mathrm{Ta}$ PAC in cubic zirconia-yttria alloys. A heuristic efg distribution approximation is required in our fitting procedures for these materials. Since we cannot presently relate this distribution to microscopic interactions, we discuss only the high-temperature relaxation parameter, which is not affected by the efg distribution. Computer simulation experiments are presently underway to 
understand the frequency distribution found in this work.

\section{Discussion of PAC Results}

A. ${ }^{111} C d$ in Tetragonal $Z r_{1-x} M_{x}$ Oxide

For $M=N b$ and $x=0.005$, the spectrum is well-fitted by a static axiallysymmetric efg with minor broadening by the random $N b$ and interstitial oxygen atoms present in the alloy. The interaction frequency is approximately one half of that computed by a simple point-ion model that ignores local lattice relaxation. A ratio of approximately two was found previously for ${ }^{181} \mathrm{Ta}$ PAC in tetragonal zirconia[1,9]. The efg is proportional to the tetrahedral distortion parameter $z$ of the near-neighbor oxyger shell[10]. We interpret these data as implying that $z$ is increased by substitution of $T a$ for $Z r$ and decreased by $C d$ substitution.

We note that there is no evidence of time-dependent efg interactions in the $N b$-dol ed samples. This is expected, since the $N b$ content is sufficient to ensure that the oxygen vacancy concentration is negligible.

For undoped zirconia and for $Y$ - and $I n$-doped samples, there is definite evidence of mobile oxygen vacancies. The relaxation parameter $\lambda$ is measureably larger than zero in all samples. The most striking feature of Fig. 2 is that the interaction frequency is strongly composition- and temperature- 
dependent. This arises because $\omega_{1}$ is a time average of a frequency $\omega_{0}$ affecting the ${ }^{111} C d$ nucleus while no vacancy is present, and a frequency $\omega_{A}$ that is the time iverage while vacancies are trapped. We emphasize that $\omega_{A}$ is not the instantaneous frequency caused by the vacancy-induced efg, rather $\omega_{A}$ is zero if the lattice is cubic, a circumstance found for ${ }^{111} \mathrm{Cd} \mathrm{PAC}$ in ceria at high temperature. If $\omega_{0}$ and $\omega_{A}$ can be determined, then the interaction frequency is $\omega_{1}=f \omega_{A}+(1-f) \omega_{0}$, and $f$, the trapping probability, can te determined readily. $\omega_{0}$ is the interaction frequency measured for $N b$-doped samples and is determined accurately. Unfortunately, we are unable to measure $\omega_{A}$ directly and must infer it from the data.

If the free vacancy concentration is c, the trap erergy is $E_{A}$, and the number of trap sites around $C d$ is $\mathrm{N}$, then the trapping probability at an isolated $C d$ atom is

$$
f(c, T)=\left[(1 / N c) \exp \left(-E_{A} / k_{B} T\right)+1\right]^{-1} .
$$

We have assumed that only one vacancy can be trapped at a time. The effect on $\mathrm{c}$ of the radioisotopes is negligible. If we neglect trapping of vacancies by dopants, then we expect $c=c_{0}+x / 4$. Here $c_{0}$ is the vacancy concentration caused by structural defects or by dopants introduced by impurities 
in the stock solution, impurities in the radioactive tracer solution, and other impurities introduced during processing. We expect $c_{0}$ to be roughly the same for all samples, and it is found to be about $350 \mathrm{ppm}$.

Under these assumptions, we have fitted the data on all undoped and $Y$ - and In-doped samples to dietermine $E_{A}, \mathrm{~N}, \omega_{A}$, and $c_{0}$. Both the PAC spectral-fit model and the equations above reiating the frequencies and relaxation to microscopic properties of the system are rigorously valid only in the limits as $c_{0}$ and $x$ approach zero. General considerations of the microscopic physics as well as a careful parametric analysis[4], show that these equations are valid only for $c_{0}+x / 4<10^{-3}$. Consequently we have fitted only data from $x=0$ to $x=0.003$. If $c_{0}$ is assumed to be the same for all samples, we find $E_{A}=0.45 \pm 0.03 \mathrm{eV}, N=23 \pm 2, c_{0}=380 \pm 60 \mathrm{ppm}$, and $\omega_{A}=0$ within statistical uncertainty. If the model is fitted to the entire composition range, $E_{A}$ converges to approximately $0.8 \mathrm{eV}, \mathrm{N}$ is smaller. and $\omega_{\dot{A}}$ is large and strongly temperature-dependent. The fitted curves then have large systematic deviation's from the experimental data.

Although we have no reason to question the analysis of the low- $x$ data, we insert a note of caution. The broad convergence range leaves considerable uncertainty. If independent information were available for $E_{A}, \mathrm{~N}$, or $\omega_{A}$, 
then the other two parameters could be determined with more confidence. Consequently, we propose a physically-satisfying scenario that is suggested by the above numbers and then use this scenario to determine an accurate value for $E_{A}$. We propose thial $N=24$ and that $\omega_{A}=0 . N$ is 24 if the vacancy is trapped at a second neighbor position, as has been suggested previously[11]. As we discuss below, there is also good evidence for second neighbor oxygen-vacancy trapping by In in $\mathrm{CeO}_{2} . \omega_{A}=\{$ also has a simple, natural physical explanation. Zero average frequency in presence of a trapped vacancy means that the efg at the $C d$ nucleus is determined only by the vacancy and that there is a negligible effect due to the lattice tetragonal distortion. The vacancy decouples the local structure from the tetragonal lattice, so the $C d$ neighborhood is no different than it would be in a cubic lattice with a vacancy trapped nearby. The fitted curves in Fig. 2 arise from this restricted model. The best-fit value for $E_{A}$ is $0.44 \pm 0.03 \mathrm{eV}$.

In this "motionally narrowed" temperature range, nucloar relaxation is dominated by efg fluctuations due to oxygen vacancies trapped by the $C d$ and hopping from trap site to trap site. In this case, $\lambda=f(c, T) \lambda_{0}(T) . \lambda_{0}(T)$ is the relaxation parameter due to a trapped vacancy and is proportional to 
$\tau_{h}$, the vacancy hopping time among trap sites.

$$
\tau_{h}=\frac{1}{\nu_{a}} e^{E_{h} / k_{B} T}
$$

So the activation energy associated with $\lambda_{0}$ is the energy barrier between trap sites. $\nu_{a}$ is the attempt frequency, and is of order $10^{14} / \mathrm{s}$. Analysis of the $\lambda$ data will be discussed elsewhere $[8,4]$. The barrier height, $E_{h}$, is approximately $0.8 \mathrm{eV}$.

B. ${ }^{111} \mathrm{Cd}$ in Undoped $\mathrm{CeO}_{2}$

Fig. 1 shows the ${ }^{111} C d$ PAC spectrum for an undoped ceria sample. The figure is an excellent example of an unbroadened, non-relaxed single site spectrum for a nucleus subject to an axially-symmetric electric field gradient. This is somewhat surprising, since the efg at a cerium atom should be zero in this cubic lattice. The non-zero efg is readily explained by the presence of a nearby trapped oxygen vacancy. Several PAC measurements have been made on $N b$ - and $Y$-doped ceria to text this hypothesis. The experiments on these doped samples have not been completed, but preliminary data show that a significant concentration of oxygen vacancies is present in these samples and support the trapped-vacancy hypothesis.

The fundamental interaction frequency is $50.6 \times 10^{6} \mathrm{~s}^{-1}$. The frequency 
calculated using the simple point jon model with no laitice relaxation is $258 \times 10^{6} s^{-1}$ for a first neighbor vacancy and $37 \times 10^{6} s^{-1}$ for a second neighbor. The experimental frequency is much too low to be caused by interaction with an oxygen vacancy trapped at a first neighbor position, but it could certainly be caused by a second-neighbor vacancy.

The PAC spectrum is qualitatively similar to Fig. 1 between the lowest temperature we have measured, $77 \mathrm{~K}$, to approximately $300^{\circ} \mathrm{C}$. The frequency is independent of tenperature, but there are some minor temperature dependent line-broadening effects that we do not yet fully understand. Above $150^{\circ} \mathrm{C}$ nuclear relaxation demonstrates that the trapped vacancies are beginning to hop at a detectable rate.

The PAC apectra are qualitatively different at temperatures above $300^{\circ} \mathrm{C}$ where the vacancy hopping rate becomes large. All data in this range can be fitted excellently by assuming a fluctuating efg due to oxygen vacancies in a cubic lattice[7]. $\lambda$ is shown in Fig. 4(a). It clearly has an exponential dependence on $1 / T$. The activation energy is approximately $0.55 \mathrm{eV}$. However, this is probably not the hopping barrier $E_{h}$. We suspect that the oxygen chemical potential is pinned by rare-earth impurity trapping and that the $\lambda$ activation energy may be a sum of $E_{h}$ and other energies that determine 
the "free" vacancy concentraticn. PAC experiments on doped ceria. now in progress may determine these energies.

C. ${ }^{181} \mathrm{Ta}$ PAC in cubic $Z r_{1-x} Y_{x}$ oxide

Analysis of the PAC relaxation for these materials in terms of a microscopic model is complicated because all these samples are concentrated alloys. The $T a$ probe is 5 -valent, so it repels vacancies rather than traps them as 2and 3-valent dopants do. Under reasonable assumptions about effects of siteaveraging, we expect the ${ }^{181} \mathrm{~T}$ a relaxation parameter $\lambda$ to be dominated by motion of oxygen vacancies hopping among sites in the bulk. For the usual simple model in which one assumes a concentration $\mathrm{c}$ of free vacancies, $\lambda$ is proportional to $c \tau_{h}$. The $\lambda$ activation energy $E_{\lambda}$ is shown in fig. $4(\mathrm{~b}), E_{\lambda}$ is independent of composition within experimental error. These data are compared to the activation energy of ionic conduction $E_{\text {cond }}[12]$, which does have a strong dependence on composition. We note that, within this free-vacancy model, the conductivity is proportional to $c / \tau_{h}$. To the extent that $c$ varies as $\exp \left(-E_{v} / k_{E} T\right), E_{\lambda}=E_{h}-E_{v}$, whereas $E_{\text {cond }}=E_{h}+E_{v}$. 


\section{Acknowledgements}

We thank Mr. John $\mathrm{Oh}$ and Mr. Randall Lundquist for technical assistance. This research has been supported in part by USDOE Contract DE-FG06-85ER45191. 


\section{References}

[1] H. Jaeger, John A. Gardner, John C. Haygarth, and R. L. Rasera, "Structural characterization of high temperature zirconia ceramics by perturbed angular correlation spectroscopy," J. Amer. Ceramics Soc. $69,458-463(1986)$

[2] H. Frauenfelder and R. M. Steffen, in Alpha,Beta, and Gamma Spectroscopies, Ch XIX, ed. K.Siegbahn, North Holland Press, Amsterdam, 1965.

[3] H. Jaeger, John A. Gardner, H. T. Su, and R. L. Rasera, "A microcomputer-controlled perturbed angular correlation spectrometer," Review of Scientific Instruments, 58, 1694-1698 (1987).

[4] H.T.Su, W. E. Evenson, and John A. Gardner, " ${ }^{111} C d$ PAC study of lightly-doped zirconium oxide," to be submitted to Hyperfine Interactions.

[5] A. Abragam, Principles of Nuclear Magnetism Oxford University Press, Oxford, UK, 1961. 
[6] W. E. Evenson, A. G. McKale, H. T. Su, and John A. Gardner, "PAC Perturbation Factor for a spin $5 / 2$ nucleus subject to a rapidlyfluctuating efg," Hyperfine Interactions, in press.

[7] William E. Evenson, John A. Gardner, Ruiping Wang, Han-Tzong Su, and Alex G. McKale, "PAC analysis of defect motion by Blume's stochastic model for $I=5 / 2$ electric quadrupole interactions," Hyperfine Interactions, in review.

[8] H. T. Su, John A. Gardner, W. E. Evenson, and J. A. Sommers,"Oxygen vacancy hopping and trapping in doped tetragonal zirconia," to be submitted to Phys. Rəv. B.

[9] H. Jaeger, PhD Thesis, Oregon State University, 1987(unpublished)

[10] H. J. F. Jansen and John A. Gardner, "Total energy calculation for ZrO2," Physica B 150, 10-18 (1988).

[11] C. R. A. Catlow, A. V. Chadwick,G. N. Greaves, L. M. Maroni, J. Amer. Ceram. Soc. 69, 272 (1986). 
[12] S. Ikeda, O. Sakurai, K. Uematasu, N. Mitzutani, and M. Kato, "Electrical Conductivity of Yttria-stabilized Zriconia Single Crystals," J.Mater. Sci. 20, 4593 (1985). 


\section{Figures}

Figure 1: Example of an "ideal" PAC spectrum, ${ }^{111} \mathrm{C} d$ in ceria at $150^{\circ} \mathrm{C}$. (a) Schematic representation of the potential energy distortion around a $C d$ probe nucleus caused by a nearby vacancy. A trapped vacancy in the second neighbor shell causes an efg, which otherwise would be zero in this cubic crystal. The nuclear energy is affected by the relative orientation of the nuclear spin direction with respect to the axis between the probe nucleus and the vacancy. This model is believed to explain the PAC data of this figure. (b) the experimental time spectrum obtained directly from raw data. $A_{2}$ is an effective anis stropy parameter, approximately -0.10 here, and $G_{2}(t)$ is a measure of the perturbation caused by interaction with an electric field gradient. (c) The Fourier transform of the time spectrum. The solid curve in part (b) is a computer fit to a s. ngle site model for a randomly-oriented polycrystalline sample with an unbroadened static efg interaction. The curve in (c) is the Fourier transform of the solid curve in (b) with the same window used for transforming the raw data. The inset in (c) shows the relationship between the efg-split nuclear energy levels for an $I=5 / 2$ nucleus and the three frequencies in the Fourier transform. 
Figure 2. PAC fundamental frequency $\omega_{1}$ for ${ }^{111} C d$ PAC in tetragonal $Z_{r_{1-x}} M_{x}$ oxide. The points are labeled by $x$ and the identity of $M$ for the sample. Where data from more than one sample of a given $x$ and $M$ are shown, the separate samples are labeled (a), (b), etc. in the legend. The solid lines are computer fits discussed in the text for which fitting parameters are $N=24, \omega_{A}=0$, and $E_{A}=0.44 \mathrm{eV}$. The fitted total oxygen vacancy concentration for each line is shown in ppm. The low-temperature deviation from the fitted line for $x=0.005$ (solid circles) is attributed to vacancy trapping by dopants. Such effects make analysis for larger $x$ difficult.

Figure 3. relaxation parameter $\lambda$ for ${ }^{111} C d \mathrm{PAC}$ in undoped ceria.

Figure 4. (a) Relaxation parameter $\lambda$ for ${ }^{181}{ }^{1} a$ PAC in cubic $Z r_{r_{1-x}} Y_{r}$ oxide (b) Activation energy $E_{\lambda}$ determined from data of (a). For comparison, ionic conductivity activation energies $E_{\text {cond }}$ are also shown. 

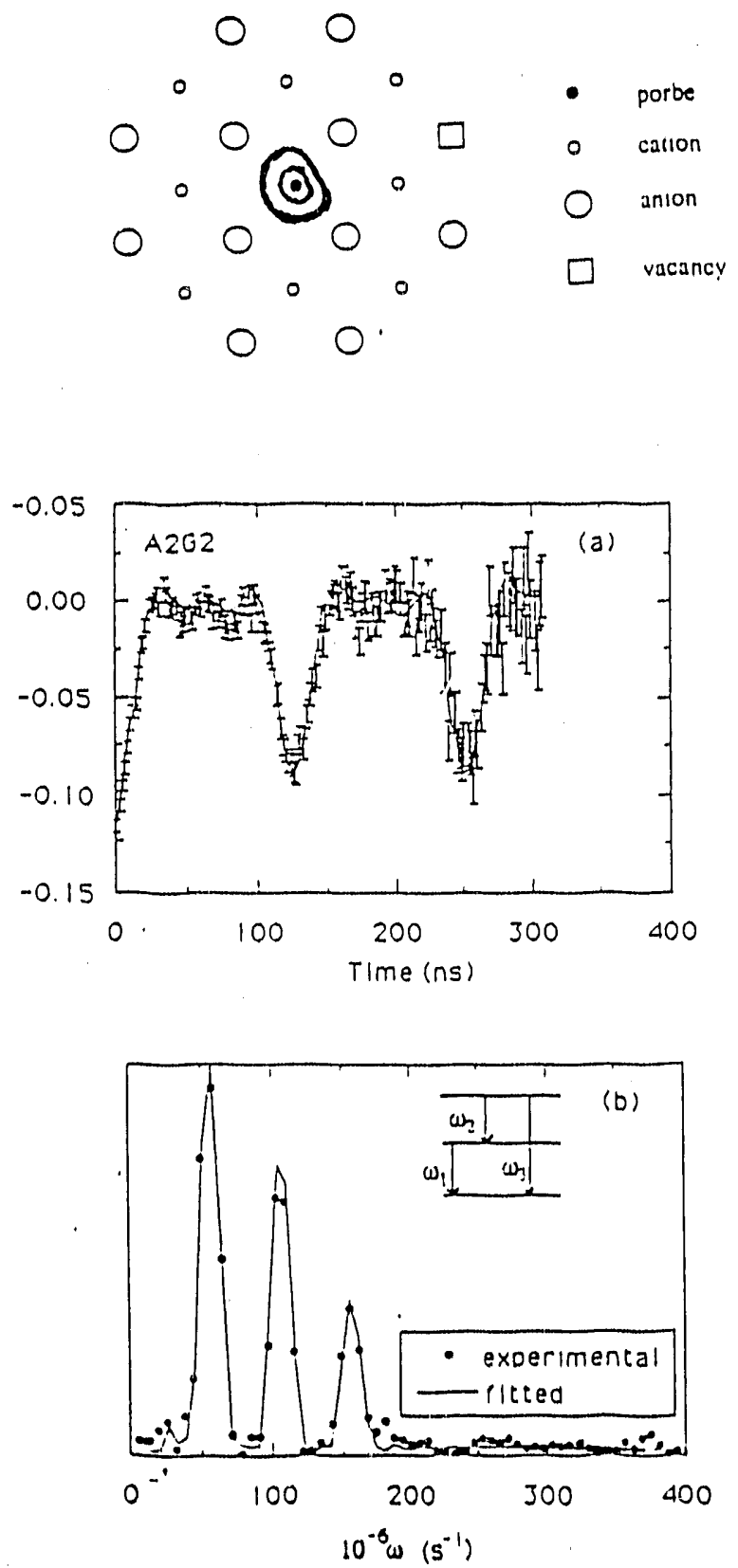


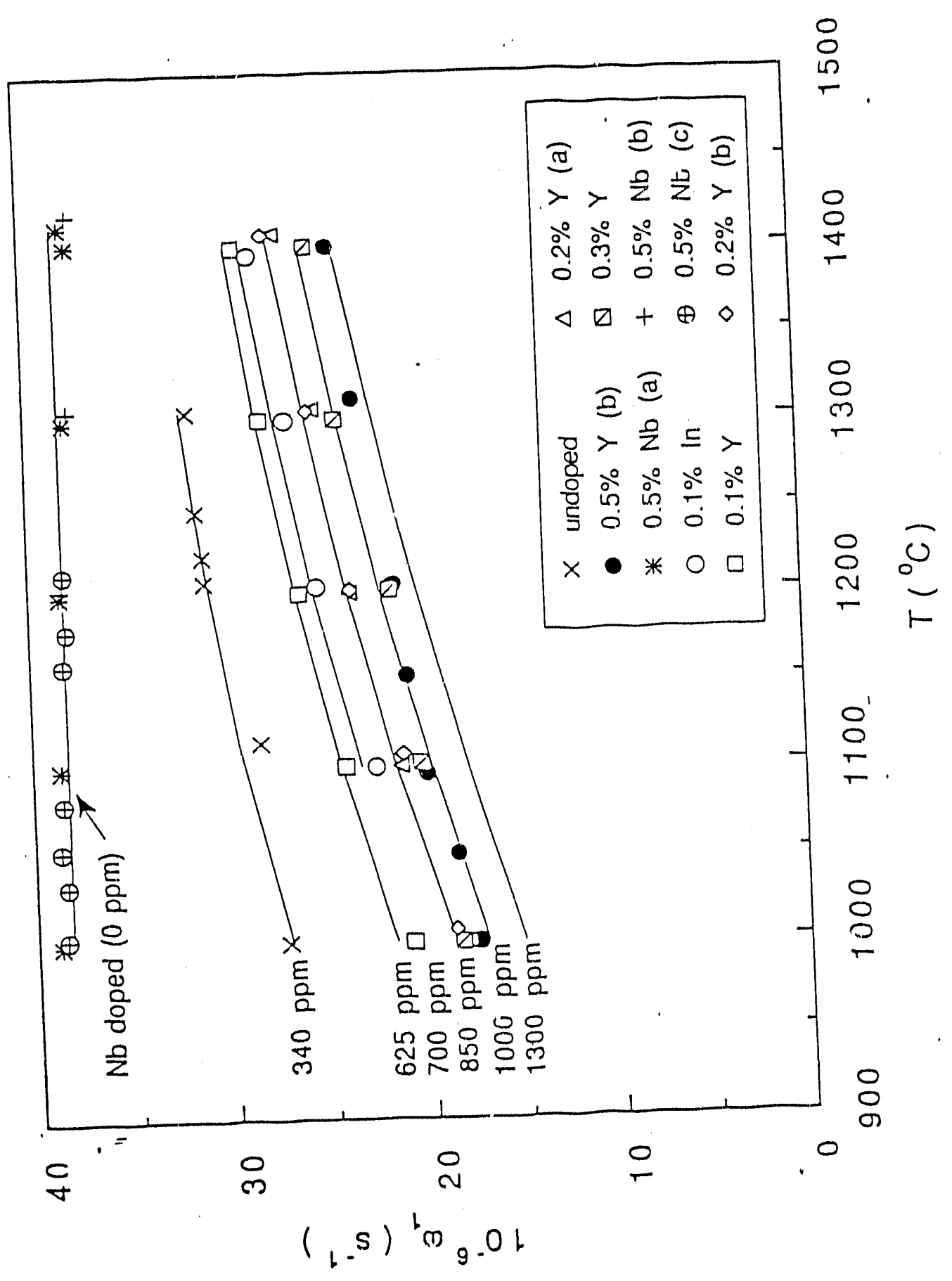




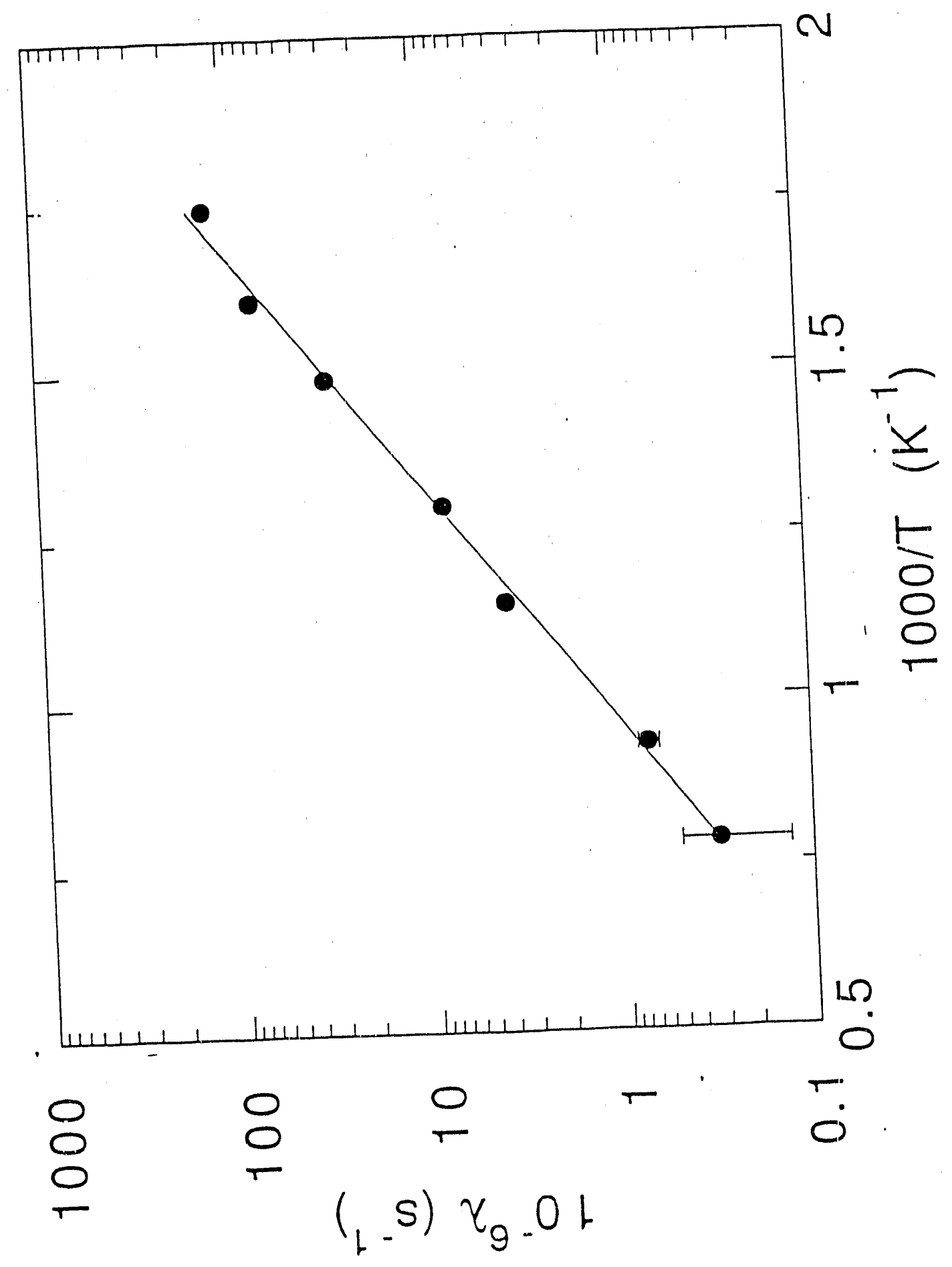

Fi:3 

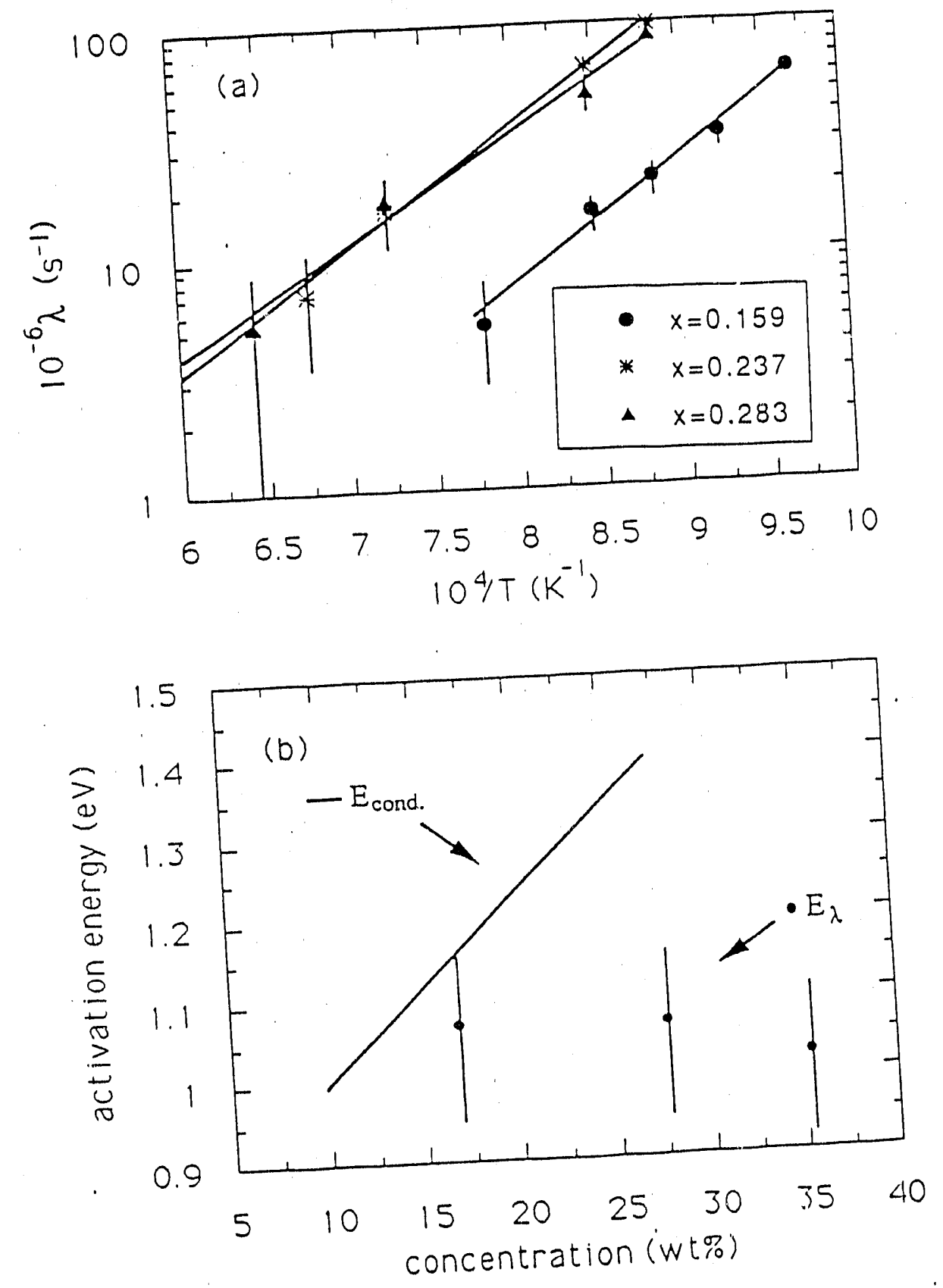

$F,: C$ 

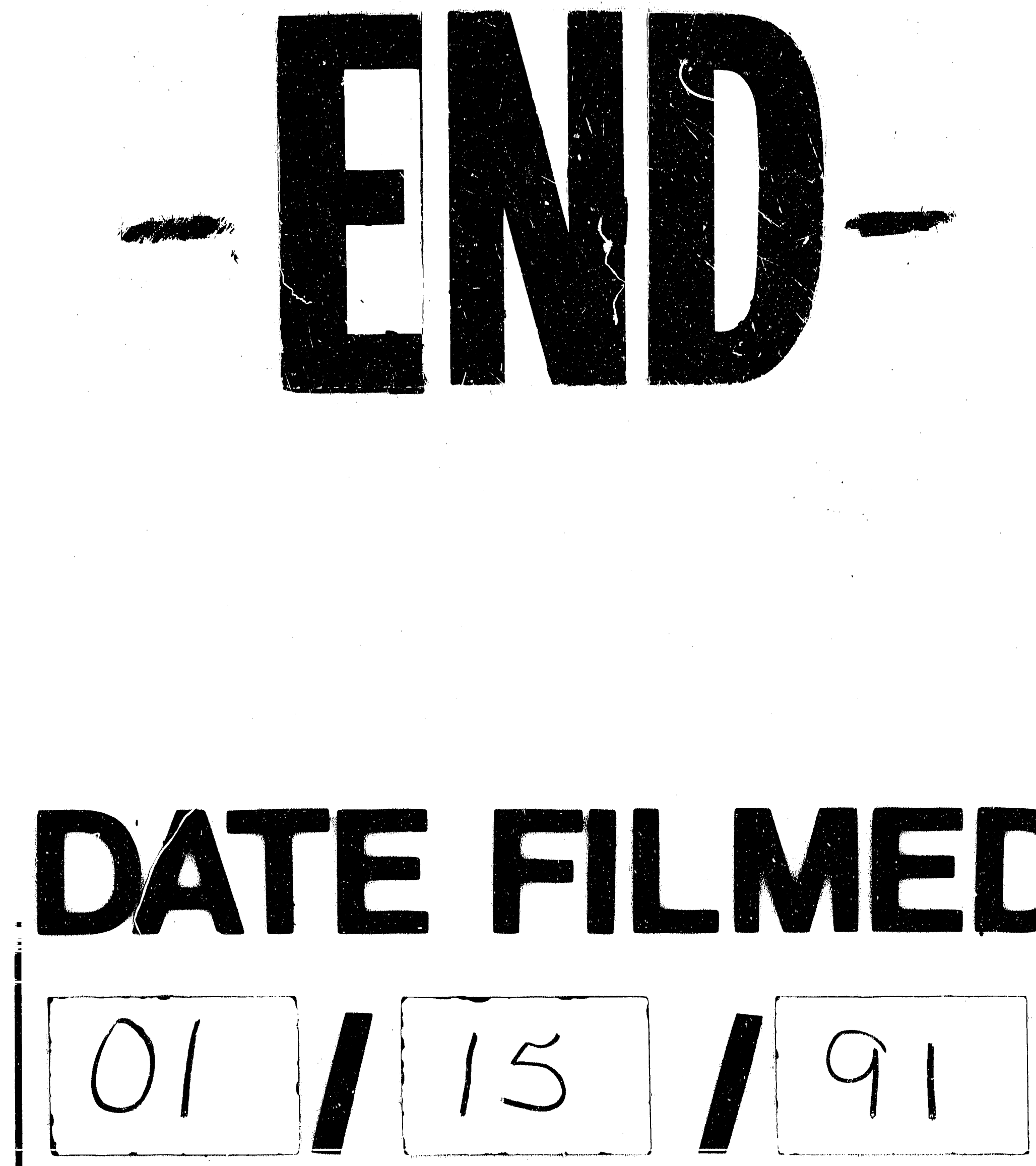
$\mathbf{E}$ 